\section{Cahiers d'ethnomusicologie}

Anciennement Cahiers de musiques traditionnelles

$7 \mid 1994$

Esthétiques

\title{
L'esthétique musicale de Sunda (Java-Ouest)
}

Aesthetics in Sundanese Music

\section{Wim Van Zanten}

Traducteur : Isabelle Schulte-Tenckhoff

\section{OpenEdition}

\section{Journals}

Édition électronique

URL : http://journals.openedition.org/ethnomusicologie/1377

ISSN : 2235-7688

Éditeur

ADEM - Ateliers d'ethnomusicologie

Édition imprimée

Date de publication : 31 décembre 1994

Pagination : 75-93

ISBN : 2-8257-0503-9

ISSN : $1662-372 X$

\section{Référence électronique}

Wim Van Zanten, «L'esthétique musicale de Sunda (Java-Ouest) », Cahiers d'ethnomusicologie [En ligne], 7 | 1994, mis en ligne le 03 janvier 2012, consulté le 21 avril 2019. URL : http://

journals.openedition.org/ethnomusicologie/1377

Ce document a été généré automatiquement le 21 avril 2019

Tous droits réservés 


\section{L'esthétique musicale de Sunda (Java- Ouest) ${ }^{*}$}

Aesthetics in Sundanese Music

Wim Van Zanten

Traduction : Isabelle Schulte-Tenckhoff

\section{Introduction}

1 L'aire culturelle de Sunda, à l'ouest de Java, comporte une population de vingt-cinq à trente millions d'individus partageant une même langue et une même tradition musicale. Toutefois, en raison de la nature complexe et hiérarchisée de la société sundanaise, la position de chacun d'entre eux peut varier énormément, de même que l'appréciation qu'il est susceptible de réserver à tel ou tel genre musical. Ceux-ci sont nombreux, allant de la musique rurale de type rituel à la musique urbaine, en passant par la celle de l'élite et celle, moins raffinée, des couches populaires.

Il serait ainsi malavisé de concevoir une esthétique commune à tous les Sundanais. A chaque genre musical correspond une manière d'interpréter la réalité sociale. Certains groupes de la société sundanaise ne connaissent guère le genre musical propre à un autre groupe. Cette société est nettement moins homogène que celle des Kaluli par exemple, décrite par Feld (1988). Il faut donc éviter des généralisations abusives. Le caractère à la fois d'unicité et d'hétérogénéité de la culture sundanaise est bien illustré par un célèbre poème, Laut Kidul : « En réalité, les différentes descriptions symboliques (sindir) sont une ; leur langage est un; ce n'est que l'interprétation qui diffère » (van Zanten 1989 : 206).

3 Dans cet article ${ }^{1}, j^{\prime}$ 'examinerai quelques aspects généraux de l'esthétique sundanaise ; plus spécifiquement, j'aborderai les principes esthétiques du genre vocal connu sous le nom de tembang Sunda (Cianjuran), qui est une sorte de musique de chambre. Le chanteur soliste est accompagné par une grande cithare et une flûte de bambou, et parfois aussi par une petite cithare au registre plus aigu. Dans les chansons basées sur le système tonal appelé 
saléndro ${ }^{2}$, une vièle à deux cordes remplace la flûte de bambou. L'élite considère le genre tembang Sunda comme la « carte de visite » de la musique sundanaise ; elle en est fière.

4 Après avoir discuté le point de vue selon lequel la musique représente une forme d'ascèse dans la société, nécessaire au bien-être de ses membres, j'aborderai des questions d'un ordre plus technique, notamment l'importance de la hauteur absolue du son. Je toucherai également au problème de la préférence accordée à la musique vocale plutôt qu'instrumentale, et à l'utilisation de microphones à Sunda. En guise de conclusion, je me pencherai sur les notions d'ordre et de désordre en musique. Quand on traite de l'esthétique musicale à Sunda, il faut en considérer les aspects sonores dans leur relation avec les aspects cognitifs. La musique est la culture: c'est une façon d'organiser l'expérience humaine, et d'exprimer des principes sociaux. Parler de musique signifie donc parler d'ordre social.

\section{La pratique musicale comme ascèse}

Dans la culture sundanaise, le son organisé ou les mouvements de danse sont généralement considérés comme la forme externe (lahir) de l'art musical ou chorégraphique. Le vrai sens (la forme interne, batin) de l'un comme de l'autre réside dans la tentative de comprendre l'essence de la vie, la femme et l'homme, la société. Certains Sundanais voient même dans la pratique musicale une voie mystique menant à l'union avec les ancêtres et les dieux. Les musiciens et les danseurs créent un contexte et une atmosphère qui permettent au spectateur de méditer sur lui-même et sur son univers.

6 Normalement, une telle méditation ou une telle ascèse (tapa) se pratique individuellement, souvent au sommet d'une montagne ou au cimetière. Pourtant, il existe d'autres formes d'ascèse. D'après le manuscrit sundanais Sanghyang siksakdang karesian, écrit sur des feuilles de palmier en 1518, c'est-à-dire avant l'extension de l'islam aux hautes terres de Sunda, il faut suivre l'exemple de ceux qui s'acquittent convenablement de leurs tâches dans la société. Une longue liste les énumère (Atja \& Saleh Danasasmita $1981: 9,35)$ : ministres, conseillers, peintres, forgerons, marionnettistes (medu wayang), musiciens (kumbang gending, musiciens, instrumentistes?; voir le commentaire dans van Zanten 1989: 33), entre autres. Selon le texte en question, ces personnages occupés à toutes sortes de tâches sont « tous disponibles pour servir le roi. Il convient de suivre leur exemple parce qu'ils pratiquent l'ascétisme dans le royaume » (tapa di nagara).

7 L'un de mes informateurs, Pandi, un musicien de tembang Sunda (Cianjur, septembre 1982), me disait que «la contemplation n'implique pas nécessairement d'escalader concrètement la montagne. La phrase «escalader la montagne» peut avoir une signification symbolique. L'interprétation du tembang Sunda selon les règles de l'art équivaut à «l'escalade de la montagne des arts » ». Fondamentalement, ce point de vue récent porte le même message que le texte de 1518 : l'art est important pour la société et peut être décrit comme tapa di nagara, c'est-à-dire l'ascétisme sans solitude. Saleh Danasasmita et Jatisunda (1986:3-7) ont décrit les activités du groupe minoritaire des Baduy à Java-ouest (dont la population est actuellement de cinq mille individus) comme tapa di nagara ou tapa di mandala: une utilisation appropriée de l'environnement pour assurer leur subsistance tout en sauvegardant le bien-être de l'univers, sans mettre en péril la vie d'autrui et sans s'enrichir. 
8 L'univers sundanais englobe les ancêtres (karuhun) qui ont contribué à forger la tradition. La quête des racines de cette tradition partagée dans la pratique de la musique, du théâtre ou de la danse est une manière d'aborder la société d'aujourd'hui. De nos jours, la tendance à valoriser le poids de la tradition est peut-être devenue plus marquée qu'elle ne l'était il y a cinquante ans (voir aussi van Zanten 1993 : 150). Dans le tembang Sunda, les musiciens les plus jeunes ont parfois le sentiment que cette attitude les empêche d'expérimenter et de s'écarter des sentiers battus. Je doute qu'ils se rendent compte du nombre de transformations que ce genre a subies tout au long du $\mathrm{XX}^{\mathrm{e}}$ siècle. L'éternel débat entre les musiciens de tembang Sunda et le public sur la bonne manière d'interpréter le genre et les possibilités de le modifier peut être considéré comme le tapa di nagara façon moderne, c'est-à-dire la méditation sur soi, sur la société sundanaise et sur l'univers.

\section{Hauteur et qualité des sons}

9 A l'ouest de Java, les sons graves sont généralement associés aux couches sociales supérieures et les sons aigus aux couches inférieures. Kusumadinata relie tout cela au système chiffré qu'il a élaboré : les tons à fréquence élevée (nada alit en sundanais, litt. "petits tons ») de l'octave sont représentés par de petits chiffres, les tons graves (nada ageng, «tons larges ») par de grands chiffres. L'échelle descendant de l'aigu au grave est transcrite ainsi : 123 4 5. Selon Kusumadinata (1969: 18-19), cela s'explique par le fait que « Si Marhaen [dénomination forgée par l'ancien président Sukarno pour se référer aux pauvres] est un petit homme ayant un statut inférieur, tandis que le ministre est un personnage important ou un grand homme, ayant un statut élevé ». Ainsi les chiffre expriment-ils le statut des notes, qui va en augmentant, des sons à haute fréquence aux sons à basse fréquence.

10 Le marionnettiste du théâtre sundanais recourt à différentes hauteurs de son pour reproduire les voix des personnages. Les plus raffinés (lungguh) «se meuvent lentement et parlent d'une voix basse, fluide »; les personnages cultivés mais fiers (ladak) «parlent d'une voix claire et se meuvent assez rapidement pour indiquer leur nature " (Foley 1979 : 33-34). De même, le conteur de carita pantun choisit la hauteur du son et la rapidité d'élocution d'après le personnage auquel il se réfère. Par exemple, dans l'histoire Lutung Kasarung, le dieu Guru Minda parle à travers le barde Enjum d'une voix basse et calme, et la princesse Purbararang parle d'une voix aiguë et traînante, «larmoyante» (enregistrement Enjum 1989).

11 Les ensembles de gamelan utilisent aussi ces procédés de variation de la hauteur du son et de la vitesse d'exécution. S'il est vrai que tous les instruments sont nécessaires à une bonne représentation musicale, une importance structurale plus grande revient aux instruments du registre grave par rapport à ceux du registre aigu. Les premiers n'émettent que quelques tons; et plus le registre est élevé, plus la densité sonore augmente. Dans le tembang Sunda également, la petite cithare aiguë (kacapi rincik) est facultative, alors que la cithare au son plus grave (kacapi indung), dont la densité sonore est la moitié de celle de la petite cithare (dans les chansons mesurées), est indispensable.

Les genres musicaux de Sunda sont aussi classés d'après la hauteur du ton de référence (diapason : barang) propre à chaque genre. La hauteur de ce ton est représentée par la longueur d'une flûte de bambou munie de six trous de jeu : «mesure » (ukuran) 61 signifie 
le ton produit par une flûte d'une longueur de $61 \mathrm{~cm}$. Genre le plus prestigieux, le tembang Sunda possède le barang le plus bas, équivalant à fa. Des genres plus populaires et moins prestigieux, comme le gamelan saléndro (utilisé par exemple pour accompagner le théâtre de marionnettes) et kacapian (chansons populaires avec accompagnement de cithare) ont un barang qui se situe autour de sib. Le barang du gamelan degung, qui était mieux valorisé socialement que le gamelan saléndro, équivaut à lab.

Dans mon analyse de l'accord des cithares dans le tembang Sunda, j'ai montré que le barang utilisé dans les années trente était nettement plus aigu, oscillant entre sol et sib. Par exemple, dans la chanson «Bayubud» (Bajoeboed) interprétée par le chanteur Radén Sanoesi et aussi par la chanteuse Résna, sur disque 78 tours (disques Sanoesi 1930?; Résna 1930 ?), la note de référence barang équivaut approximativement à sib. La même chanson figure sur une cassette commercialisée, interprétée cette fois-ci par Iyus Wiradiredja (1981). Dans cet enregistrement réalisé lors du concours de chant DAMAS à Bandung, les 13 et 14 février 1981, on annonce sur la cassette que la «mesure » (ukuran) utilisée est 61, ce qui implique un barang de fa. En moyenne, le barang du tembang Sunda a diminué de 400 cent $^{3}$, tandis que le barang des autres genres ne s'est guère modifié (van Zanten 1986 : 90-91; $1989: 116-18$ ).

14 J'ai suggéré que cette modification du barang pouvait s'expliquer comme une tentative de souligner l'importance culturelle du genre. En recourant à un barang plus grave, on place le tembang Sunda à l'écart des autres genres. Avant 1900, le tembang Sunda était pratiqué exclusivement par l'élite, alors de de nos jours, tout un chacun peut l'apprendre, du moins en principe. Le fait de baisser la hauteur absolue des chansons de tembang Sunda est peut-être une réaction (inconsciente ?) pour contrer ce processus de démocratisation entamé au début de ce siècle. Plutôt qu'une tentative d'enrayer ce processus, le prestige considérable du tembang Sunda fut exprimé en termes proprement musicaux, c'est-à-dire par la hauteur absolue du son des chansons.

15 Nano S. est un célèbre compositeur de chansons populaires sundanaises recourant à l' instrumentarium sundanais (degung kawih, kacapian), qui enseigne à l'école secondaire de musique de Bandung (SMKI). Il conteste mon hypothèse selon laquelle le registre grave des chansons actuelles de tembang Sunda s'accorde bien avec la classification des genres en fonction de leur importance sociale (Nano 1993). D'après lui, les chansons de tembang Sunda ont une hauteur de son plus grave en raison de l'influence de feue Ibu Saodah (Saodah Harnadi Natukusumah 1922-1981), qui fut pendant longtemps chanteuse à Radio Bandung (RRI). Elle avait une voix plutôt grave et chantait certainement dans un registre plus bas que la «mesure 61 » (barang de fa), recourant souvent à la «mesure 64-65 » ( barang oscillant entre mib et ré). Ce style a sans doute exercé une influence sur la hauteur du son. Il arrive qu'un individu transforme la société. Mais sa préférence pour le registre grave ne pouvait être acceptée et devenir la norme dans les concours de chant que si elle était considérée comme approprié et conforme aux notions générales d'esthétique, lesquelles comprennent justement une hiérarchie des hauteurs du son.

16 Cette hiérarchie et son lien avec le statut social se retrouve aussi dans le langage parlé. Dans un article fort intéressant, Ohala (1983) discute de l'utilisation translinguistique de la hauteur du son: "On constate, à travers les langues et les cultures, des similitudes remarquables dans les fonctions signalétiques de la hauteur du son de la voix ». Après avoir résumé l'état actuel de la recherche en la matière, notamment le rapport entre les voix humaines et celles de certains animaux (comme le grognement menaçant ou les gémissements soumis du chien), l'auteur conclut: «Ces modèles peuvent s'expliquer en 
admettant que la communication vocale humaine recourt à un "code des fréquences " selon lequel l'espèce toute entière associe les émissions vocales aiguës à la petite taille (de celui qui les produit) et à l'absence de menace, tandis que les émissions vocales graves évoquent la grande taille de celui qui les produit et ses desseins menaçants » (Ohala 1983 : 1). Il discute également de l'usage possible de la hauteur du son dans le langage et les associations qu'il suggère :

«Considérons tout d'abord la hauteur du son par rapport au type de phrase. La hauteur du son communique immédiatement la taille, mais en dernière analyse elle véhicule sans doute de nombreuses autres significations par association. Une hauteur de son grave signifie probablement non seulement "grand" mais aussi agressif, péremptoire, confiant, dominant, auto-suffisant, et ainsi de suite. A côté de "petit", la hauteur de son aigu peut signifier rassurant, soumis, subordonné, ayant besoin de l'assistance ou de la bonne volonté du récepteur, etc. Celui qui pose une question dépend implicitement des autres pour obtenir l'information recherchée. Pour le signaler au moyen du code de fréquences, il utliserait une hauteur de son aiguë. En revanche, celui qui énonce une affirmation déclare implicitement sa certitude ou son auto-suffisance - du point de vue de l'information - et utiliserait donc, s'il recourait au code de fréquences, une hauteur de son grave » (Ohala 1983 : 8-9).

Si cette description confirme les résultats de mes recherches sur la musique sundanaise, il faut néanmoins garder présent à l'esprit qu'il s'agit là de généralisations. Par exemple, bien que les langues indonésiennes tendent à marquer les questions par une élévation de la hauteur du son à la fin de la phrase, cela n'est pas toujours le cas, puisqu'on peut également poser des questions selon un modèle descendant, comme dans Sekarang mau ke mana? (Où veux-tu aller maintenant ?). Un aspect crucial est qu'Ohala indique les mêmes associations que celles que j'ai pu identifier dans la musique de Sunda: grand-grave et petit-aigu.

Van Bezooijen (1993) aborde, quant à elle, les facteurs qui déterminent la hauteur moyenne du son dans le langage, comme le physique, l'âge, le sexe, le statut socioéconomique et l'origine régionale de celui qui parle. Il s'agit là de déterminants à long terme (ou extra-linguistiques), contrairement à l'utilisation de glissements dans la hauteur de son pour indiquer des aspects linguistiques comme l'accent, l'interrogation ou l'affirmation, ou encore à des aspects para-linguistiques comme le stress, l'émotion ou l'attitude (van Bezooijen 1993 : 166-67). Pour le sujet qui nous occupe, le rapport entre l'âge et le statut socio-économique d'une part, la hauteur moyenne du son dans le langage parlé d'autre part revêt un intérêt particulier. Sur la base de données empiriques au sujet de cinq groupes de femmes, van Bezooijen (1993: 175) n'a pas pu confirmer un rapport entre hauteur du son et statut socio-économique. Par contre, sur la base de données relatives à 140 Hollandais et Hollandaises adultes, elle a pu dégager un rapport entre la hauteur moyenne du son dans le langage (45 secondes de parole semi-spontanée) et l'âge (van Bezooijen 1993 : 172-73). Tant pour les hommes que pour les femmes, quoique à un degré moindre dans ce dernier cas, la hauteur moyenne diminue légèrement jusqu'à l'âge de quarante ans, puis elle reste constante jusqu'à l'âge de soixante ans, pour augmenter systématiquement à nouveau entre soixante et soixante-dix ans. Van Bezooijen suppose qu'en dehors de facteurs purement physiologiques, ce rapport est déterminé par des traits de personnalité comme les a relevés Ohala (1983) : la dominance et l'indépendance. Dans les cultures occidentales, les personnes âgées ne jouent en général qu'un rôle relativement subordonné et dépendant, doté de peu de prestige social. Ce changement 
particulièrement manifeste pour les hommes au moment de la retraite s'exprime par une élevation de la hauteur moyenne du son.

D'une manière générale, l'influence exercée par la culture sur la hauteur moyenne du son dans le langage parlé vient confirmer mon hypothèse que les gens de Sunda associent la hauteur du son musical au prestige et au statut social; le plus important étant qu'il semble y avoir un rapport, encore que celui-ci puisse prendre différentes formes selon les cultures. Ainsi, dans le théâtre d'ombre balinais, c'est plutôt la voix aiguë qui est considérée comme raffinée et dotée de prestige (Vonck s.d.).

\section{Simple et raffiné}

A Sunda, l'opposition est radicale entre ce que l'on considère comme raffiné, cultivé et fin (lemes) et ce qui est simple, grossier, rude, abusif (kasar). Il existe par exemple divers niveaux de langage en sundanais, allant du raffiné au vulgaire. La plupart des mots courants du sundanais sont kasar. Toutefois, si la personne à laquelle on s'adresse de vive voix ou par écrit doit être traitée avec respect, parce que est plus âgée ou d'un rang plus élevé, certains termes kasar sont remplacés par des termes lemes. Les termes du niveau immédiat (sedeng) sont aussi utilisés dans de telles circonstances, mais seulement si celui qui parle se réfère à sa propre situation ou action, ou à celle d'un tiers. Ces trois niveaux de langage ne sont que les plus importants. J'ai fréquemment entendu les personnes âgées se plaindre que la jeune génération ne sache plus utiliser correctement les niveaux de langage du sundanais.

Les différents genres musicaux peuvent aussi être placés entre ces deux pôles, c'est-à-dire entre lemes et kasar; à l'intérieur d'un même genre, certains sons peuvent être décrits comme raffinés, d'autres comme simples. Le tembang Sunda est considéré comme une musique raffinée (lemes), tandis que des genres comme jaipongan, kacapian et le théâtre de marionnettes (wayang golék) sont plutôt simples (kasar).

A l'intérieur du tembang Sunda, certaines phrases mélodiques dans le registre grave, interprétées d'une voix basse et rauque comme si elles étaient chuchotées (ngahéas), sont considérées comme très cultivées. Mon principal informateur, Uking Sukri, a décrit une manière semblable de jouer des passages dans le registre grave sur la grande cithare et la flûte de bambou, en les qualifiant de «chuchotement» (gerentes), ou de "paroles douces et lentes » (nyarios lalaunan). A l'opposé de ce type de jeu retenu et cultivé, on trouve le jeu soutenu, aigu et strident de la flûte de bambou appelé gelik, auquel on recourt généralement dans les chansons Dedegungan du répertoire. D'après Uking Sukri, ce sont notamment ces phrases aiguës et soutenues de la flûte de bambou qui évoquent la mélancolie. Les sons graves et doux (de l'instrument degung qui fait partie du gamelan degung, à savoir six gongs suspendus verticalement) évoquent la stabilité et le confort, tandis que les sons plus aigus et chargés d'émotion de la flûte de bambou rendent les gens tristes (chanson de tembang Sunda « Bébér Layar »; voir van Zanten 1989 : 212) :

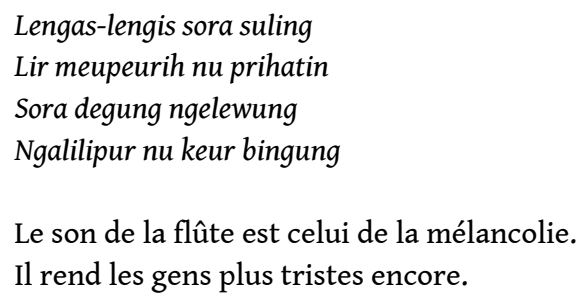


Le son du degung est profond

Et réconforte ceux qui sont attristés. genres musicaux comportent du chant, et il existe de nombreux termes se référant à «chanter", "chant» ou "musique vocale» (van Zanten 1989: 13-36). Il y a des exceptions : dans les campagnes, on rencontre le jeu de la guimbarde (karinding), des flûtes de bambou(bangsing, elét, suling kumbang) et le pilage cérémoniel du riz (gondang, gendék); dans les villes, la seule musique purement instrumentale est le genre appelée kacapi-suling, deux cithares et une flûte de bambou. Néanmoins, la musique sundanaise est essentiellement vocale, qu'il s'agisse du prestigieux tembang Sunda ou du genre très populaire appelé jaipongan (accompagné de danse), ou encore de formes traditionnelles comme la récitation carita pantun et les différents ensembles de gamelan. Cette abondance de musique vocale par comparaison à la musique instrumentale est indéniable. Mais il est plus difficile d'en préciser les raisons. Voici une première tentative allant dans ce sens.

Un cas intéressant est le gamelan degung, purement instrumental à l'origine. Ce gamelan typiquement sundanais s'est probablement développé entre 1800 et 1850 dans l'entourage des souverains de Sunda. Dès le début du XVIII e siècle, les souverains de Java-ouest s'étaient considérablement enrichis (Sutherland 1973: 125-26). L'introduction du « système Preanger " par les colonisateurs hollandais leur avait permis d'amasser des fortunes en contraignant leurs sujets à leur fournir certains biens. Il n'est dès lors guère surprenant qu'ils aient tenté de légitimer leur position en développant de prestigieuses formes d'art comme le tembang Sunda Cianjuran et le gamelan degung, différentes des genres de cour pratiqués dans le centre de Java (van Zanten 1993 : 150).

29 est servi de plus en plus pour accompagner le chant, surtout celui des femmes. De nos jours, le gamelan degung strictement instrumental est rarement interprété - tendance parallèle à l'évolution qu'ont connue au $\mathrm{XX}^{\mathrm{e}}$ siècle les ensembles de gamelan (saléndro) accompagnant le théâtre de marionnettes (wayang). Depuis les années cinquante, la chanteuse soliste occupe une place de plus en plus importante dans l'ensemble, après le conteur et meneur de l'ensemble (dalang). Dans un séminaire organisé récemment à Bandung en hommage à la chanteuse Upit Sarimanah (1928-1992), le 29 octobre 1992, il 
est apparu qu'on lui avait accordé beaucoup plus d'importance dans le théâtre de marionnettes qu'au conteur lui-même. Avant 1900, le genre en question ignorait la chanteuse soliste. Mais depuis, celle-ci s'est également imposée dans le tembang Sunda. De nos jours, ce sont principalement des femmes qui chantent le tembang Sunda, tandis que le jeu instrumental reste l'apanage des hommes. La même tendance est apparue dans le gamelan degung.

On peut s'interroger sur les causes de cette évolution. Peut-être résulte-t-elle de l'émancipation des femmes : elles ont été graduellement acceptées comme chanteuses sur les scènes de cette région dominée par l'islam. Jadis, la chanteuse et la danseuse (ronggéng ) avaient mauvaise réputation; on les considérait souvent comme des prostituées. Actuellement, les femmes jouent un rôle central dans la transmission des textes chantés, voire même dans la récitation de versets coraniques (pangajian) sur cassettes commercialisées.

31 Mais il ne faut pas non plus oublier qu'avant 1900 le chant a toujours joué un rôle important. Mises à part les danseuses et les chanteuses, il y avait par exemple les chansons du marionnettiste du théâtre wayang et le barde des contes pantun. Pourquoi les Sundanais (et les Javanais) n'ont-ils qu'à peine développé une musique instrumentale, à la différence des Indiens avec le sitar et les tabla? Rappelons que la musique purement instrumentale du kacapi-suling est une exception, et qu'elle est marginale aux yeux des Sundanais, parce que jouée presque exclusivement dans les grands hôtels de Bandung en fin d'après-midi, pour divertir les touristes ou hommes d'affaires étrangers. Dans le cadre de sa politique de développement d'une industrie touristique, le gouvernement indonésien encourage les ensembles de kacapi-suling à se produire dans les hôtels. D'autre part, le genre se perpétue principalement grâce aux cassettes commercialisées, car les Sundanais ne songent pas souvent à engager un tel ensemble pour animer une réception de mariage ou un rituel de circoncision, préférant plutôt un ensemble vocal, notamment un ensemble interprétant le tembang Sunda qui, tout en étant musicalement apparenté au kacapi-suling, est doté d'un prestige nettement plus élevé que celui-ci.

Une raison expliquant l'abondance de la musique vocale par rapport à la musique instrumentale est qu'aux yeux de la plupart des Sundanais, la technique de jeu requise par la seconde ne revêt guère d'intérêt. Il est rare, même pour un musicien professionnel, de s'exercer seul au jeu de passages difficiles. En règle générale, il n'y a pas non plus de répétitions ou, s'il y en a, celles-ci se font avec l'ensemble au complet au cours de sessions informelles l'après-midi ou le soir. La musique est l'expression de la pensée - ou du "verbe intérieur» - et, de ce fait, elle est plus une affaire d'inspiration plutôt que de virtuosité. Ce qui compte, c'est l'esprit dans lequel on joue, non la perfection technique. La technique ne se rapporte qu'à la manifestation externe (lahir) ; la perfection technique est atteinte lorsque la disposition interne (batin) est bonne, et non l'inverse ${ }^{4}$. Les instruments sont des outils pour faire de la musique et, par voie de conséquence, ils sont davantage liés au monde externe que le chant. Les chanteurs étant de toute évidence dotés d'un statut plus élevé que les instrumentistes, il n'est pas abusif de penser que le chant est beaucoup plus proche de l'essence musicale que le jeu instrumental.

Pour les Sundanais, le son (sora) en tant que force créatrice comprend la parole et la musique: il apporte la vie. Comme le dit aussi l'Évangile selon saint Jean: "Au commencement était le Verbe " (voir aussi van Zanten 1989: 96). L'opposé du son n'est pas le silence, mais la mort, car le silence peut comprendre la pensée, le «verbe intérieur ", comme l'exprime aussi la philosophie védique (Essers 1952: 128). Les 
Sundanais semblent en convenir, en particulier en ce qui concerne le chant. Suanda (1985 : 93) remarque à propos du gamelan de Cirebon (région culturelle située entre Sunda et Java), qu'« une représentation de gamelan pendant laquelle il n'y a pas assez de chant est considérée comme "morte » ». La communication par le chant recourt au mode musical autant qu'au mode linguistique. Le langage fait défaut aux animaux, et c'est le plus puissant des divers sons - ou des diverses forces créatrices - de l'univers humain.

Dans le tembang Sunda, un type de voix quasi idéal est ampuh, « modeste et bien contrôlé » (van Zanten 1989: 180). C'est la voix idéale des nobles. Le terme ampuh comporte une autre signification, à savoir «doté de pouvoirs exceptionnels », lorsqu'il se rapporte notamment au poignard (keris), aux formules magiques ou aux guérisseurs (dukun). Ampuh peut également signifier "efficace ", et c'est dans ce sens qu'il s'applique par exemple à un médicament ou à une méthode d'enseignement (Eringa 1984 : 19).

Le chant semble être perçu comme étant plus «beau » que la musique instrumentale, car il est plus intériorisé et associé aux puissances qui contrôlent la vie. Peut-être ces idées sont-elles encore assez répandues dans la culture de Sunda, ce qui expliquerait la préférence accordée à la musique vocale plutôt qu'instrumentale.

Dans ce contexte, il convient de mentionner l'utilisation de microphones par les musiciens (Fig. 1). Dans le tembang Sunda - et d'autres genres -, l'attitude générale semble être de favoriser l'usage de microphones et d'une sonorisation quand ils sont disponibles, spécialement pour les chanteurs, quelle que soit la dimension de la salle. Même des pièces de quatre mètres sur cinq sont souvent sonorisées. La manière dont certains chanteurs de tembang Sunda se servent du microphone rappelle l'usage qu'en font les chanteurs pop : ils le maintiennent tout près de la bouche et produisent des effets spéciaux en variant la distance. 
Fig. 1 : Chant avec sonorisation par l'interprète de tembang Sunda Énah Sukaénah pendant une fête de mariage au domicile de la mariée.

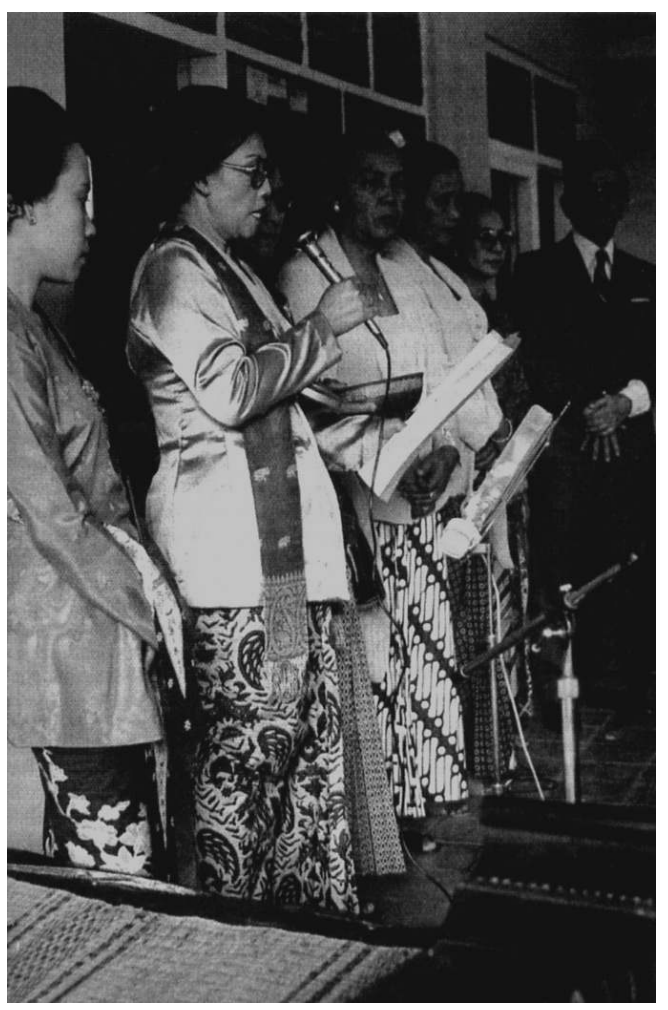

Photo : Wim van Zanten, 1981. chambre », me laisse perplexe. Qu'est-ce qui motive le choix des musiciens, même dans des situations qui ne semblent pas l'exiger? La fascination qu'exercent les moyens modernes est-elle plus puissante que les notions traditionnelles d'esthétique? Sans doute l'idée que le « verbe intérieur » l'emporte sur le son réellement produit est influencée à son tour par l'évolution et les possibilités de la technique. Les musiciens de tembang Sunda disent que l'utilisation de microphones leur permet de mieux faire entendre les ornements vocaux délicats. En chantant fort, on a nettement plus de peine à les exécuter. Comme je l'ai déjà dit, le chant fort n'est guère apprécié; le choix et l'interprétation convenables des ornements vocaux sont considérés comme l'essence même du genre tembang Sunda. Il semble donc logique de donner la priorité à leur bonne émission. La sonorisation ne se rapporte qu'aux aspects externes de la musique; le chanteur étant ainsi libre de se concentrer sur l'essence, à savoir les ornements vocaux, plus proches du "verbe intérieur ». De même, certains chanteurs m'ont expliqué que les instruments d'accompagnement (kacapi, suling) ne sont pas très importants à leurs yeux; d'après eux, on pourrait tout aussi bien se servir d'un piano ou d'une guitare.

Jusqu'en 1985, de nombreux enregistrements commerciaux sur cassette contenaient des effets d'écho supplémentaires obtenus électroniquement. Cette manipulation des enregistrements a diminué ces dernières années. Cela signifie peut-être que la fascination initiale pour les possibilités techniques de l'enregistrement a quelque peu diminué - ce qui pourrait également se répercuter sur le recours à la sonorisation.

Cahiers d'ethnomusicologie, 7 | 2011 


\section{Les émotions et l'aspect féminin de la communication humaine}

Aux dires des Sundanais, la musique est chargée d'émotions; elle évoque souvent des sentiments de tristesse et de mélancolie. Dans la vie quotidienne, il ne faut pas montrer trop ouvertement ce que l'on ressent; c'est spécialement mal vu chez les hommes. La pratique musicale fournit l'occasion de communiquer ses sentiments. Une chanson du tembang Sunda dit : "Je me suis réveillé au son de la flûte de bambou/Mon cœur est lourd de chagrin». Dans un conte du carita pantun il est dit que «la flûte de bambou et la guimbarde se lamentent» (Atjeng Tamadipura 1970: 116). «Jouer du grand rebab» ( ngarebab jangkung) signifie « être triste » (Kamus Umum Basa Sunda 1976 : 423, 512). Dans un autre conte de carita pantun (Taswan 1961: 91-92), on dit que le singe lutung (la réincarnation de Ratu Bungsu Karma Jaya) achète un gamelan. "Puis le lutung se mit à pleurer, en écoutant le son du gamelan. C'est ainsi que, jusqu'à ce jour, lorsque la progéniture du Prophète Adam entend le gamelan, elle fait l'expérience de ses émotions ».

Les Sundanais se décrivent comme étant généralement de bonne humeur et joyeux dans la vie quotidienne. Les sentiments de tristesse et, d'une manière générale, les émotions fortes sont principalement communiqués dans un contexte musical. On pourrait dire que, dans la pensée sundanaise, la musique représente les aspects féminins de la communication humaine. Les sons du rebab et de la guimbarde (karinding) sont comparés à la voix féminine. Le plus souvent, le chant est une affaire de femmes. Apparemment elles sont aujourd'hui les médiatrices privilégiées dans ce processus de communication relatif à la part « ineffable » de la vie quotidienne, qui, elle, est contrôlée par les hommes.

Dans la plupart des cultures d'Indonésie, les rapports entre les générations jouent un rôle crucial. C'est souvent le cadet qui gouverne, l'aîné étant l'ascète ou le conseiller dépourvu d'un pouvoir réel. En analysant les principes culturels de l'ethnie baduy établie à l'ouest de Java, Louis Berthe (1965: 222) a souligné que «la substance de l'aîné a pour attribut principal la fémininité, celle du cadet au contraire, la virilité ». L'ascétisme, les aspects réflexifs et interprétatifs des arts, sont l'apanage de l'aîné. La remarque de Berthe vient donc confirmer notre idée que les arts représentent les aspects féminins de la communication.

\section{Ordre et désordre}

Dans la musique sundanaise, les notions d'ordre et de désordre sont très présentes. Tout d'abord, je donnerai quelques exemples provenant du tembang Sunda, pour montrer ensuite que les concepts musicaux sont étroitement liés aux notions relatives à l'ordre social.

Dans le tembang Sunda, la principale division à l'intérieur des chants passe entre ceux exécutés en rubato (mamaos) et ceux qui sont mesurés (kawih ou panambih). Les chants sont toujours interprétés par séries de deux, trois ou quatre, en commençant par les chansons mamaos et en terminant par celles appelées chants kawih. Cette évolution allant de l'exécution en rubato à celle en rythme mesuré est semblable au mode d'interprétation d'un râga en Inde. Du point de vue idéologique, les chansons mamaos l'emportent. Il arrive, sur les cassettes commercialisées dans les années 1970, que les chansons mesurées 
ne soient même pas mentionnées - en dépit du fait que, du point de vue musical, on semble souvent prendre plus de plaisir à l'écoute de ces mêmes chansons.

En se plaçant à l'intérieur d'une série de chansons de tembang Sunda, on pourrait expliquer cette évolution comme le passage d'un «désordre» relatif (rubato) à un " ordre » strict (mètre). Dans le jeu rubato, la grande cithare commence par émettre des cascades sonores mettant en valeur les notes autour desquelles la chanson est organisée. On dit que cela sert à «familiariser » le chanteur avec le système modal utilisé (pélog, sorog ou saléndro). Ce style de jeu de la cithare se poursuit lorsque le chanteur entonne son chant; on l'appelle pasieupan, "harmoniser", du mot sieup, "en harmonie, doté de proportions justes, accordé ». Une fois que le chant a commencé, la cithare et la flûte doivent suivre le chanteur et lui donner les signaux d'entrée au bon moment.

Dans les chants mesurés, les deux cithares sont appelées à produire un rythme très régulier. Le tempo doit rester stable, et le jeu doit être organisé avec précision pour arriver aux notes pivots. Ce sont là des critères qui s'appliquent surtout au jeu de la petite cithare. Sur la grande cithare, la main droite produit des formules régulières (notes aiguës), mais ce rythme strict est "contrebalancé » par les syncopes qu'émet la main gauche. Cette dernière partie est aussi appelé «battre le tambour ». L'effet musical est appelé géngsot, c'est-à-dire: légère déviation, différence dans la durée; l'un de mes informateurs l'a comparé à un pantalon dont les deux jambes n'auraient pas la même longueur. Le jeu à contretemps peut être considéré comme une sorte de désordre destiné à faire ressortir l'organisation stricte du rythme et rendant la musique de type kawih plus intéressante. D'une manière similaire, dans les années 1980, les joueurs de la petite cithare kacapi rincik ont commencé à utiliser occasionnellement des triolets - pratique qui peut également être vue comme une tentative d'éviter un rythme par trop régulier.

Bien que la chanson obéisse à une métrique régulière, le flûtiste et le chanteur continuent à suivre plus ou moins le style rubato. Toutefois, leurs phrases musicales sont reliées par les sons de "gong " terminant une phrase dont la durée est généralement de huit temps (env. MM 48-52). Un chanteur peut « contrebalancer » cette conclusion sur la dernière note de « gong » en devançant légèrement les deux cithares.

En 1985, j'ai assisté à une représentation où des danseurs de penca silat accompagnaient la musique de tembang Sunda (Fig. 2). Au commencement, durant la phase vocale en rubato, les mouvements du danseur étaient abrupts, et il émettait des cris occasionnels. Pendant les chansons mesurées, ses mouvements devenaient plus souples. Le meneur de l'ensemble m'a expliqué qu'«il faut lutter pour atteindre au bonheur». En d'autres termes, après avoir combattu le désordre, on parvient à l'ordre, à l'harmonie et au bonheur.

$48 \mathrm{Au}$ cours d'une représentation, il arrive que le public fasse des commentaires. Dans l'enregistrement d'une soirée informelle de tembang Sunda en 1981, on entend l'exclamation "Aduh, saé» (oh, c'est bien), des rires, des gens faisant claquer leur langue : "ch", puis à nouveau "saé" (bien) pendant les parties instrumentales d'une chanson mesurée (enregistrement audio Éméh Salamah 1981: A054, A067). A la fin de la chanson, la chanteuse s'excuse, car elle ne s'est plus souvenu des paroles à un moment donné : " Punten waé. Hapunten, pabeulit», "Je regrette la confusion (concernant le texte)». Toutefois, la musique, son chant sont appelés imeut, précis, soignés. 
Fig. 2 : Danse penca silat sur une musique tembang Sunda : « il faut lutter pour atteindre le bonheur ». Danse aux gestes discrets, accompagnée d'un chant métrique.

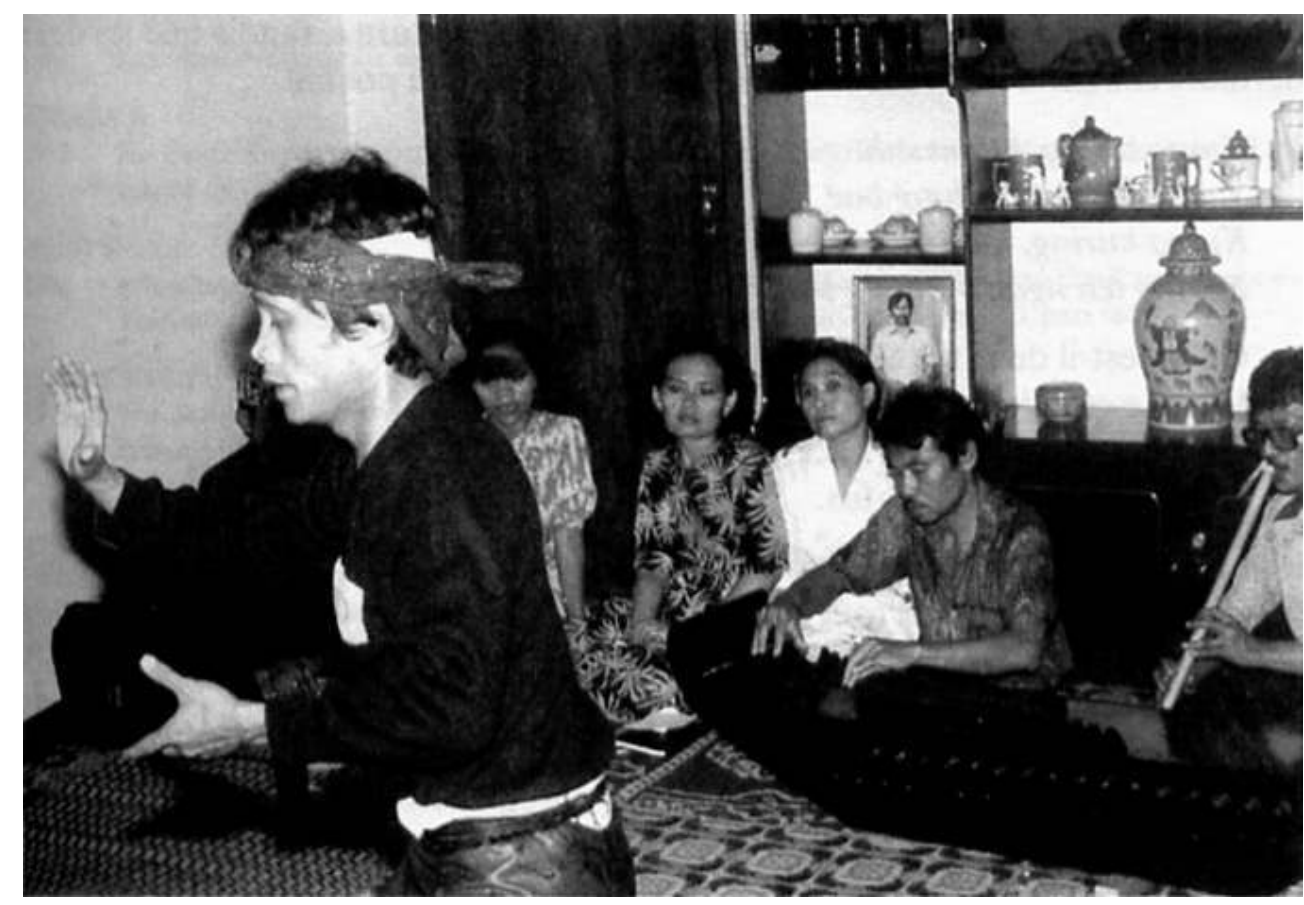

Photo : Wim van Zanten.

Un instrument de musique mal accordé peut servir de métaphore d'une perturbation de l'ordre social. Il existe divers termes pour signaler le désaccord d'un instrument : palsu, du hollandais vals; sumbang, silung and sorog. A part « désaccordé », le terme de sumbang signifie aussi «allant à l'encontre de la tradition, de la coutume», ou « inceste ». Dans le texte suivant, puisé dans un conte de carita pantun, le gong - qui est mal accordé symbolise le roi (ou la reine) qui " est en désaccord» avec ses sujets (voir van Zanten 1989 : 194 ; pour l'enregistrement du texte, voir la cassette accompagnant l'ouvrage, plage 1):
Goong pélog sosorogan
Goong sumbang pangrampogan
Paranti ngélékheun musuh
Lain éléh ku hadéna
Eléh ku sumbangna baé
Le gong pélog est désaccordé.
Un gong sonnant faux risque d'être volé,
Le moyen pour vaincre l'ennemi
Ne fera pas défaut s'il est en bon état
[Mais] il sera défaillant s'il est en désaccord.

Si les relations humaines sont fondamentalement mauvaises, l'ordre social est troublé et la société se désintègre.

L'ordre social troublé peut être représenté par différents symboles, tels le roi malade et le gong sonnant faux. Le joueur de cithare "épouse » son instrument. Ce dernier étant considéré comme son épouse, l'instrumentiste doit établir de bons rapports avec lui. Par exemple, il doit l'accorder juste pour que ce « mariage » engendre la musique. 
Il existe un célèbre poème sur une flûte désaccordée, dont la forme met en évidence une assonance entre les deux premiers et les deux derniers vers. Les deux premiers sont appelés la "peau» ou la "couverture», tandis que les deux derniers contiennent la substance ou la signification du poème.

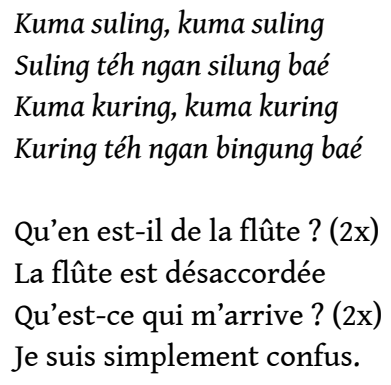

Il se peut que les deux premières lignes de ce poème soient dépourvues de signification ; dans ce cas il est tout à fait approprié d'associer la flûte sonnant faux au désordre ou à la confusion. Ce vers est fréquemment utilisé par les chanteurs dans le tembang Sunda. On le chante pendant l'interlude instrumental d'une chanson mesurée qui, elle, n'est interprétée en principe que par une femme. Il comporte des implications érotiques (voir l'enregistrement vidéo Panglawungan 1990 : V90-1, $\left.1^{\text {h }} 07^{\prime} 30^{\prime \prime}-1^{\mathrm{h}} 08^{\prime} 4^{\prime \prime}\right)$.

L'ordre social et l'ordre musical sont donc étroitement liés. Pour conclure mon article, je donnerai un exemple récent. Il y a quelques années, un célèbre joueur de flûte de bambou a divorcé de sa femme sundanaise. Il l'a quittée, elle et leurs cinq enfants, pour se rendre à l'étranger, car il était amoureux d'une de ses étudiantes étrangères et il est allé vivre avec elle. La plupart de ses collègues musiciens ont désapprouvé cette atteinte à l'ordre social. Après une année environ, il est revenu pendant quelques mois à Java-ouest pour acheter une maison à son ex-femme sundanaise. Pendant ce temps, il a joué de la flûte de bambou avec ses anciens collègues. L'un d'eux a remarqué que son jeu s'était dégradé, bien qu'il ait continué à jouer de la flûte à l'étranger. Comme les rapports sociaux avaient été perturbés, il était normal que son jeu fût également « en désaccord ».

\section{BIBLIOGRAPHIE}

ATJA \& SALEH Danasasmita, 1981, Sanghyang siksakanda ng karesian ; Naskah Sunda kuno tahun 1518 Masehi. Bandung : Proyek Pengembangan Permuseuman Jawa Barat.

ATJENG Tamadipura, 1970, Tjarita Mundinglaja di Kusumah. Bandung : Projek Penelitian Pantun. [Recité par Ki Atjeng Tamadipura (Situradja, Sumedang), supervisé par Ajip Rosidi].

BERTHE Louis, 1965, « Aînés et cadets ; l'alliance et la hiérarchie chez les Baduj (Java occidental) », L'Homme V

(3-4) : 189-223.

BEZOOIJEN Renée van, 1993, «Verschillen in toonhoogte : Natuur of cultuur ». Gramma/TTT, tijdschrift voor taalwetenschap $2: 165-79$.

ERINGA F.S., 1984, Soendaas-Nederlands woordenboek. Dordrecht-Holland : Foris. [KITLV]. 
ESSERS B., 1952, En Oudindische symboliek van het geluid. Assen : van Gorkum. [Thèse de doctorat, Université de Groningen].

FELD Steven, 1988, « Aesthetics as iconicity of style, or « lift-up-over sounding » : getting into the Kaluli groove », Yearbook for Traditional Music 20 :74-113 + cassette audio [Volume 20, part two].

FOLEY Kathy, 1979, The Sundanese wayang golek : the rod puppet theatre of West Java. Unpublished Ph.D. dissertation, University of Hawaii.

HATCH Martin, 1985, « Nyai Bei Mardusari : singer of Javanese poetry », Review of Indonesian and Malaysian Affairs $19: 113-36$.

KAMUS Umum Basa Sunda, 1976, Kamus Umum Basa Sunda. Bandung : Tarate.

KUSUMADINATA R. Machjar Angga, 1969, Ilmu seni-raras. Jakarta : Pradnja Paramita.

NANO S., 1993, Communication personnelle, 2 août 1993, au SMKI de Bandung.

OHALA John J., 1983, «Cross-language use of pitch : an ethological view ». Phonetica $40: 1-18$.

SALEH Danasasmita et Anis Djatisunda, 1986, Kehidupan Masyarakat Kanekes. Bandung :

Departemen Pendidikan dan Kebudayaan, Direktorat Jenderal Kebudayaan, Bagian proyek

Penelitian dan Pengkajian Kebudayaan Sunda (Sundanologi).

SUANDA Endo, 1985, «Cirebonese topeng and wayang of the present day », Asian Music 16-2 : 84-120.

SUTHERLAND Heather, 1973, « Notes on Java's regent families ; Part 1 », Indonesia 16 :113-47.

TASWAN, 1961, Galur pantun Ratu Bungsu Karma Jaya (Lutung Leutik). Djakarta : Dana Guru. [Récité par Taswan, Kuningan ; transcrit de l'audition du 26 au 28 juin 1958 par R. S. Wirananggapati ; introduction par R. Satjadibrata].

VONCK Henrice M., s.d., "The music of the North Balinese shadowplay ». [à paraître].

ZANTEN Wim van, 1986, "The tone material of the kacapi in tembang Sunda in West Java ", Ethnomusicology $30: 84-112$.

ZANTEN Wim van, 1989, Sundanese music in the Cianjuran style; Anthropological and musicological aspects of tembang Sunda. [Verhandelingen van het Koninklijk Instituut voor Taal-, Land- en Volkenkunde no. 140 ; avec cassette audio]. Dordrecht-Holland / Providence-U.S.A. : Foris.

ZANTEN Wim van, 1993, « Sung epic narrative and lyrical songs : carita pantun and tembang Sunda ", in : ARPS Bernard (ed.) : Performance in Java and Bali; Studies of narrative, theatre, music and dance. London : School of Oriental and African Studies : 144-161.

\section{Documents audio et vidéo}

EMEH Salamah, 1981, chant rubato (mamaos) « Madenda Sari » suivi d'un chant mesuré (kawih) « Gandrung Gunung », interprété par Emeh Salamah, accompagné par Rukruk Rukmana (kacapi indung), Uju Suhari (suling) et Edi Supratman (kacapi rincik). Soirée informelle tembang Sunda à Bandung le 3 septembre 1981. Enregistrement de l'auteur sur la cassette audio TS13, A 023-112 ; 7' 55" avec commentaire à la fin.

ENJUM, 1989, Enregistrement de carita pantun « Lutung Kasarung » par l'auteur dans la nuit du 8 au 9 novembre 1989 à Ujungberung. Cassette 89-10, A498-510.

IYUS Wiradiredja, 1981, Bayubud chanté par Iyus Wiradiredja (alias R.M. Yusuf) au concours de chant DAMAS, Bandung, du 13 au 14 février 1981. Bandung : Hidayat cassette tape S-1025. 
PANGLAWUNGAN, 1990, « Kuma suling... » comme il devrait être interprété par un chanteur en un chant mesuré « Budak Ceurik », chanté par une femme (Ottih Rostoyati). Enregistrement vidéo de la réunion des musiciens et connaisseurs de tembang Sunda (Panglawungan) aux studios de télévision TVRI de Bandung le 21 octobre 1990. Enregistrement de l'auteur sur cassette vidéo V 90-1 de 1 h 06 ' 05" à 1 h 09' 25 ".

RÉSNA, 1930?, Bayubud [Bajoeboed] chanté par Résna sur le disque 78 t. Odeon (Jab 131). Copié dans la collection du Musée Pusat, Jakarta, 1981.

SANOESI, 1930?, Bayubud [Bajoeboed] chanté par Radén Sanoesi sur le disque 78 t. Odeon (Jab 123). Copié dans la collection Musée Pusat, Jakarta, 1981.

\section{NOTES}

*. Traduit de l'anglais par Isabelle Schulte-Tenckhoff.

1. Cet article est basé sur une communication présentée au huitième Séminaire européen d'ethnomusicologie, organisé à Genève du 22 au 28 septembre 1991.

2. En sundanais, é se prononce comme le è en français, tandis que $e$ se prononce comme dans «le » en français.

3. Le cent est une unité de mesure de l'intervalle musical résultant d'une fonction logarithmique de la fraction de fréquence, telle que 100 cent $=1$ demi-ton de la gamme tempérée ( 1 octave $=1200$ cent).

4. Hatch $(1985: 119,125)$ offre des remarques similaires à propos de l'apprentissage du chant à Java central.

\section{RÉSUMÉS}

Dans la pensée de Sunda, les sons ne représentent que la forme externe de la pratique musicale. La pratique de la musique, voire des arts en général est une forme « d'ascèse dans le royaume » ( tapa di nagara), c'est-à-dire une réflexion sur soi-même et le monde environnant. Pour assurer le bien-être de la société et de ses membres, il faut que la musique, la danse, le théâtre et la poésie soient pratiqués convenablement. L'esthétique de Sunda conçoit une hiérarchie des hauteurs du son: aux notes graves revient un statut plus élevé qu'aux notes aiguës. Le chant raffiné du tembang Sunda confère beaucoup de prestige, et sa hauteur du son est inférieure à celle de genres plus «simple" comme kacapian et degung kawih. Les notes graves évoquent la stabilité et le confort, tandis que les notes plus aiguës et davantage chargées d'émotion expriment un sentiment de tristesse. Rarement la musique de Sunda est purement instrumentale. Au contraire, la musique implique généralement du chant. Une tentative est faite pour démontrer que le chant est plus valorisé que la musique instrumental, car il est plus intériorisé et associé aux pouvoirs qui dominent la vie. La musique transmet ce qui est « ineffable » dans la vie quotidienne, à savoir le domaine contrôlé par les êtres humains. L'article explore l'hypothèse que les arts représentent les aspects féminins de la communication humaine. Toute la musique de Sunda est dominée par des notions d'ordre et de désordre. Par exemple, un instrument de musique mal accordée peut servir de métaphore de l'ordre social troublé. 
In Sundanese thinking musical sounds are only the outer form of music-making. Practising music - and generally the arts - is a kind of "ascetism in the kingdom » (tapa di nagara), that is, a reflection on oneself and the world. The proper performance of music, dance, theatre and poetry is needed for the well-being of society and its members. In Sundanese aesthetics, there is a hierarchy among pitches: low notes have greater status than high notes. The refined singing of tembang Sunda has much prestige, and its pitch level is lower than the more « simple " genres like kacapian and degung kawih. Low notes are associated with stability and comfort, whereas the emotional, higher notes evoke feelings of sadness. There is not much purely instrumental Sundanese music. Most music involves singing. An attempt is made to explain that singing seems to be more highly valued than instrumental music because it is more internalised, and associated with the powers which control life. Music communicates matters « ineffable » in daily life, which is the domain controlled by men. It is argued that the arts represent the female aspects of human communication. Notions of order and disorder pervade all of Sundanese music. For instance, a musical instrument which is out of tune may be used as a metaphor for the social order being upset.

\section{AUTEURS}

\section{WIM VAN ZANTEN}

Wim Van Zanten possède une formation de physicien théorique et a enseigné les mathématiques à l'Université de Malawi de 1967 à 1971. Il s'est intéressé en parallèle à la musique du Malawi du sud. En 1971, il a commencé à enseigner à l'Institut d'études culturelles et sociales de l'Université de Leiden. De 1976 à 1979, il a donné un enseignement sur l'utilisation des statistiques en sciences sociales à l'Université de l'Indonésie, et il est l'auteur de deux livres en indonésien sur ce sujet. Mais son intérêt principal est la musique de Sunda (Java occidental). Actuellement, il prépare une méthode pour l'interprétation du tembang Sunda Cianjuran. D'autre part, il mène des recherches sur la musique des Baduy, minorité ethnique de Java ouest. Il s'intéresse en outre aux aspects anthropologiques de l'analyse musicale. 